\title{
FAAH inhibition enhances anandamide mediated anti- tumorigenic effects in non-small cell lung cancer by downregulating the EGF/EGFR pathway
}

\author{
Janani Ravi ${ }^{1}$, Amita Sneh ${ }^{1}$, Konstantin Shilo${ }^{1}$, Mohd W. Nasser ${ }^{1}$ and Ramesh K. \\ Ganju $^{1}$ \\ ${ }^{1}$ Department of Pathology, The Ohio State University, Ohio, USA. \\ Correspondence to: Mohd W. Nasser, email: Mohd.nasser@osumc.edu \\ Ramesh K Ganju, email: Ramesh.ganju@osumc.edu \\ Keywords: NSCLC, EGFR, Met-F-AEA, FAAH \\ Received: December 19, 2013 Accepted: February 19, $2014 \quad$ Published: February 21, 2014
}

This is an open-access article distributed under the terms of the Creative Commons Attribution License, which permits unrestricted use, distribution, and reproduction in any medium, provided the original author and source are credited.

\section{ABSTRACT:}

The endocannabinoid anandamide (AEA), a neurotransmitter was shown to have anti-cancer effects. Fatty acid amide hydrolase (FAAH) metabolizes AEA and decreases its anti-tumorigenic activity. In this study, we have analyzed the role of FAAH inhibition in non-small cell lung cancer (NSCLC). We have shown that FAAH and $C B 1$ receptor which is activated by AEA are expressed in lung adenocarcinoma patient samples and NSCLC cell lines A549 and H460. Since the synthetic analogue of anandamide (Met-F-AEA) did not possess significant anti-tumorigenic effects, we used Met-F-AEA in combination with FAAH inhibitor URB597 which significantly reduced EGF (epidermal growth factor)-induced proliferative and chemotactic activities in vitro when compared to anti-tumorigenic activity of Met-F-AEA alone. Further analysis of signaling mechanisms revealed that Met-F-AEA in combination with URB597 inhibits activation of EGFR and its downstream signaling ERK, AKT and NF-kB. In addition, it inhibited MMP2 secretion and stress fiber formation. We have also shown that the MetF-AEA in combination with URB597 induces G0/G1 cell cycle arrest by downregulating cyclin D1 and CDK4 expressions, ultimately leading to apoptosis via activation of caspase-9 and PARP. Furthermore, the combination treatment inhibited tumor growth in a xenograft nude mouse model system. Tumors derived from Met-F-AEA and URB597 combination treated mice showed reduced EGFR, AKT and ERK activation and MMP2/MMP9 expressions when compared to Met-F-AEA or URB597 alone. Taken together, these data suggest in EGFR overexpressing NSCLC that the combination of Met-F-AEA with FAAH inhibitor resulted in superior therapeutic response compared to individual compound activity alone.

\section{INTRODUCTION}

Non-small cell lung cancer (NSCLC) is a metastatic form of cancer which accounts for about $85 \%$ of lung cancer cases and is the primary cause of cancer related deaths in the United States. Though tobacco smoking is the major cause of lung cancer, about $15-20 \%$ of cases are attributed to non-smokers and involve the activation of various signaling pathways for tumor development [1]. Adenocarcinoma and squamous cell carcinoma, the two most common histological subtypes of lung cancer are categorized as NSCLC. Poor prognosis and chemotherapeutic resistance which may be due to modulation of key cell signaling mechanisms pose major concerns [2-3].

The cannabinoid family is categorized into endogenous cannabinoids (produced inside the body), phytocannabinoids (plant derived) and synthetic cannabinoids which activate the specific G-protein coupled receptors $\mathrm{CB} 1$ and $\mathrm{CB} 2$. CB1 receptor is mainly expressed in the brain and CNS whereas CB2 receptor is expressed in immune system [4-6]. The use of cannabinoid 
agonists as anti-cancer agents has proven successful in various in vitro and in vivo cancer models such as glioma, breast, prostate, colon, leukemia and lymphoid tumors [7-10]. They have been shown to modulate various cell survival pathways such as the extracellular signalrelated kinase (ERK), phosphoinositide 3-kinase (PI3K), p38 mitogen-activated protein kinase (p38 MAPK), protein kinase B (AKT) and ceramide pathways [1113]. Anandamide (AEA) and 2-arachidonoylglycerol (2AG) are the two well characterized endocannabinoids which are endogenous ligands for the cannabinoid receptors. Although endocannabinoids were initially studied for their neurological and psychiatric effects, there is increasing evidence of their contribution to inflammation and tumorigenesis [14-15]. AEA, which is mainly synthesized from phospholipids, is inactivated by enzyme fatty acid amide hydrolase (FAAH) mediated hydrolysis to arachidonic acid (AA) and ethanolamine (EA), whereas 2-AG is hydrolyzed into AA and glycerol [16-20]. Thus, the effects of the endocannabinoids are profoundly affected by their enzyme mediated hydrolysis. Moreover, inactivation of FAAH activity has been shown to potentiate the anti-tumorigenic effects of AEA in prostate cancer [21]. However, the exact roles of FAAH and its regulation of AEA activity have not been elucidated in the context of tumorigenicity in NSCLC. In our work, we focus on AEA, an endogenous cannabinoid agonist specific for the CB1 receptor and the effect of FAAH inhibition on the activity of AEA.

The genetic abnormalities associated with lung cancer are attributed to alterations in the signaling pathways which are targets for drug therapies. Most of these stimulatory signaling pathways are driven to a malignant phenotype characterized by uncontrolled proliferation and an apoptosis escape mechanism. Epidermal growth factor receptor (EGFR) is a family of four Receptor tyrosine kinases (RTKs) EGFR (ERBB1, HER1), ERBB2 (HER2, Neu), ERBB3 (HER3) and ERBB4 (HER4) [22-23]. EGFR dysregulation is associated with multiple cancer types including malignant transformations and metastasis [24]. EGFR overexpression and signaling pathway gene mutations play a vital role in lung tumorigenesis. Recent evidence suggests that cancer cells undergo escape mechanisms to defend against the host system by activation of alternative growth signaling pathways [25]. The cell cycle in eukaryotes is regulated by a family of cyclins and cyclin dependent kinases (CDKs), which are members of protein kinase complexes. Each complex consists of a cyclin (regulatory subunit) which binds to a CDK (catalytic subunit) to form an active cyclin-CDK complex that gets activated at various checkpoints during the cell division cycle [26-27]. Several studies indicate that cell cycle markers are mutated in most malignant cancers and might lead to Programmed Cell Death (PCD), where cells undergo suicide program
[26-28]. Apoptosis is a type of PCD which involves the activation of caspases and DNA fragmentation [29-31]. Cell cycle dysregulation and resistance to apoptosis are often attributed to abnormal EGFR signaling [22, 32]. Hence, identification of novel receptors expressed in tumor cells that target against EGFR activation will be a promising strategy against NSCLC.

In our present study, we analyzed the effect of AEA on lung tumorigenesis when FAAH is inhibited. We show that Met-F-AEA in combination with URB597 reduces NSCLC growth in vitro and in vivo. Our results reveal that the combination treatment inhibits the activation of EGFR and its downstream signaling targets. These findings suggest the possibility of exploring the components of endocannabinoid system as a novel therapeutic target for NSCLC treatment.

\section{RESULTS}

\section{Primary lung cancer tissues and NSCLC cell lines express CB1 and FAAH}

Anandamide is known to mediate its effects through cannabinoid receptor $\mathrm{CB} 1[7,30]$. Hence, the expression of FAAH and CB1 were assessed in the NSCLC cell linesA459, A549, CALU1, H460 and H1299 (Fig 1A). All the cell lines expressed FAAH and CB1 as detected by Western Blot. Also, we utilized tissue microarray (TMA) to detect levels of FAAH and CB1 in cancer patients. Eleven of fifty seven (19.3\%) lung adenocarcinomas showed high cytoplasmic expression of FAAH (Fig 1B, C). Twenty two of thirty five $(62.9 \%)$ lung adenocarcinomas showed high cytoplasmic expression of CB1 (Fig 1D, E).

\section{FAAH inhibition enhances the anti-proliferative activity of Met-F-AEA in NSCLC cell lines}

Proliferation is one of the characteristic features of cancer cells to grow and multiply [33-35]. To analyze whether inhibiting FAAH can enhance the activity of Met-F-AEA (synthetic analogue of anandamide), we evaluated the potential of Met-F-AEA in combination with FAAH inhibitor- URB597 as a possible therapeutic target. We treated the NSCLC cell lines- A549 and H460 with either FAAH inhibitor- URB597 or Met-F-AEA or in combination and observed the effects after $24 \mathrm{~h}$. MTT assay showed a significant decrease in cell viability in the combination treatment when compared to Met-F-AEA alone (Fig 2A, B). Also, we performed clonogenic assay which measures the ability of single cell to form clones. Combination treatment significantly reduced the number of colonies when compared to Met-F-AEA or URB597 alone in both the cell lines (Fig 2C, D). 

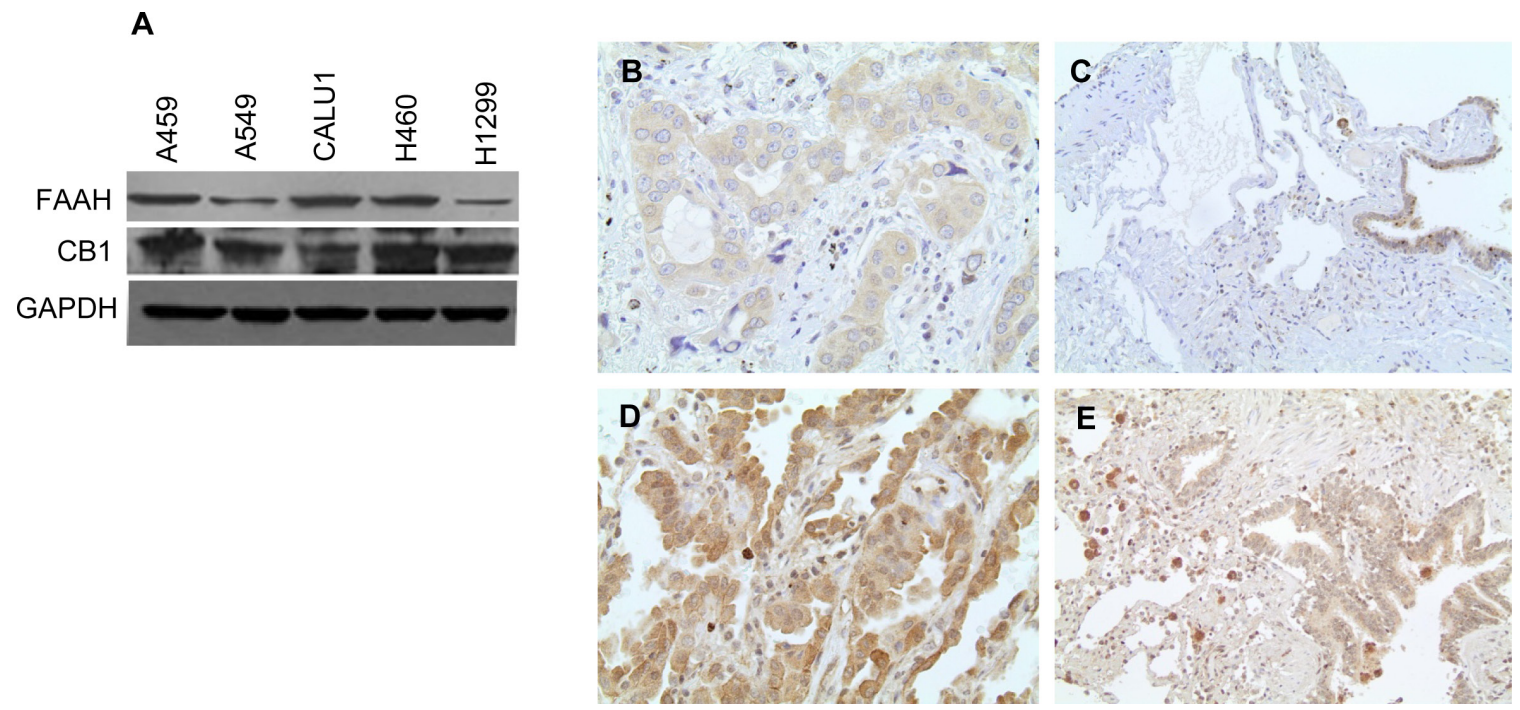

Figure 1: NSCLC cell lines and primary lung cancer tissues express CB1 and FAAH. (A) NSCLC cell lines were subjected to immunoblot analysis to determine the expression of CB1 and FAAH. GAPDH served as loading control. Representative photomicrographs of IHC staining of FAAH expression in lung adenocarcinoma (B) and respiratory epithelia (C). Representative photomicrographs of IHC staining of CB1 expression in lung adenocarcinoma (D) and respiratory epithelia (E).

A

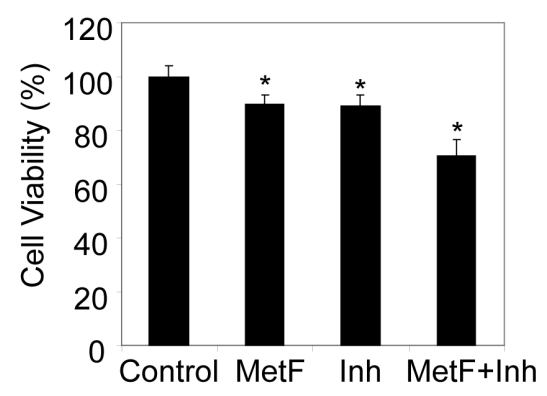

C

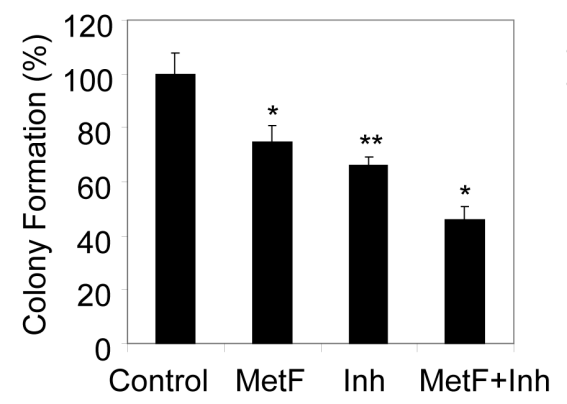

B

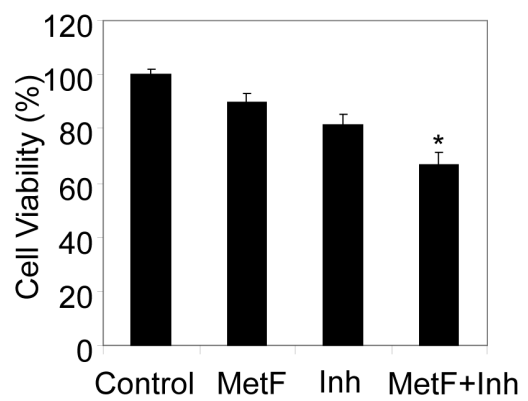

D

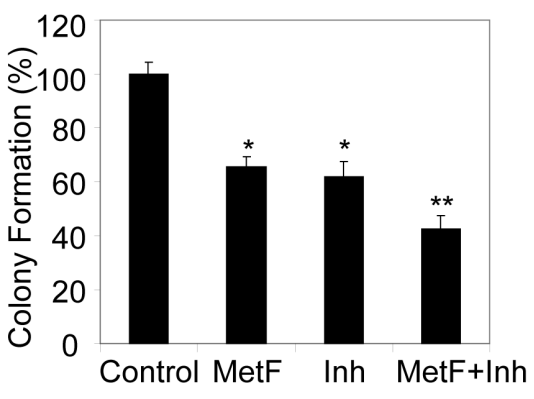

E

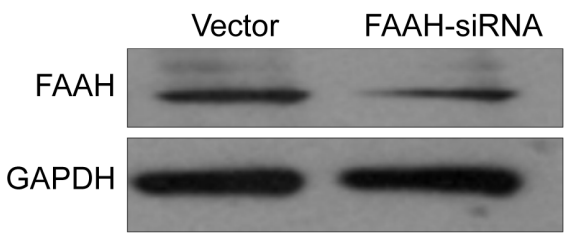

$\mathbf{F}$

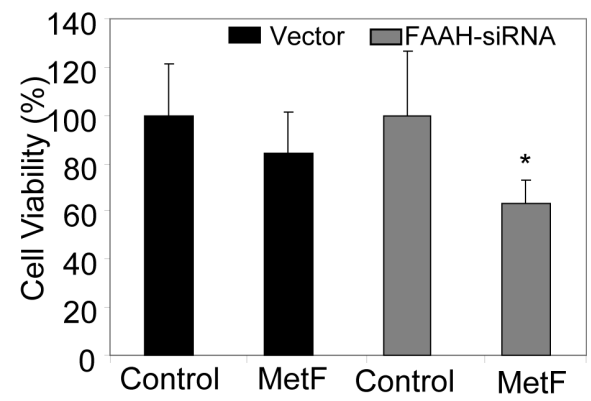

Figure 2: FAAH inhibition enhances the anti-proliferative activity of Met-F-AEA in NSCLC cell lines. A549 (A) and H460 (B) cells were serum starved for 24h and treated with control, Met-F-AEA (MetF, 10 $\mu \mathrm{M}$ ), FAAH inhibitor URB597(Inh, $0.2 \mu \mathrm{M}$ ) or MetF+Inh and analyzed for viability by MTT assay. 1000 individual A549 (C) and H460 (D) cells were plated in six well plates and subjected to colony formation assay by treating with control, Met-F-AEA (MetF, 10 $\mu \mathrm{M}$ ), FAAH inhibitor URB597 (Inh, $0.2 \mu \mathrm{M}$ ) or MetF+Inh as shown for six days. The colonies were then fixed, stained and counted. (E) H460 cells were transfected with 100pmol of either the non targeting control (vector) or FAAH-siRNA (Dharmacon) using Lipofectamine 2000 (Invitrogen) according to the manufacturer's instructions for 36h and the expression of FAAH in siRNA and non targeted cells were evaluated by Western blot. (F) H460 cells which were transfected with either FAAH siRNA or non targeted control (vector) for 36h were treated with Met-F-AEA for 24h and subjected to MTT assay. $\mathrm{P}<0.05\left(^{*}\right)$ and $\mathrm{P}<0.005\left({ }^{* *}\right)$ as calculated by Student's $t$ test. Data represent the mean $\pm \mathrm{SD}$ per experimental group. 
To enhance the effect of the endocannabinoid Met-FAEA, we used the siRNA approach to knockdown FAAH in H460 cells. To analyze the efficiency of knockdown, the transfected cells were subjected to Western Blot analysis, which showed reduced expression of FAAH (Fig 2E). Cells transfected with FAAH siRNA were more sensitive to Met-F-AEA treatment than the control siRNA transfected cells (Fig 2F). These data suggest that Met-FAEA in combination with URB597 inhibits cell growth in vitro.

\section{FAAH inhibition enhances the anti-migratory and anti-invasive activities of Met-F-AEA in NSCLC cell lines}

Migration and invasion are required for cancer cells to spread within the specific tissue and form the characteristic features of angiogenesis and metastasis [36]. The extracellular matrix (ECM) acts as a barrier towards this cell motility process. Chemoattractants like EGF provide a directed migration for cancer cells [22, $32,36]$. We further analyzed the effects of Met-F-AEA in combination with URB597 on EGF induced chemotaxis
A

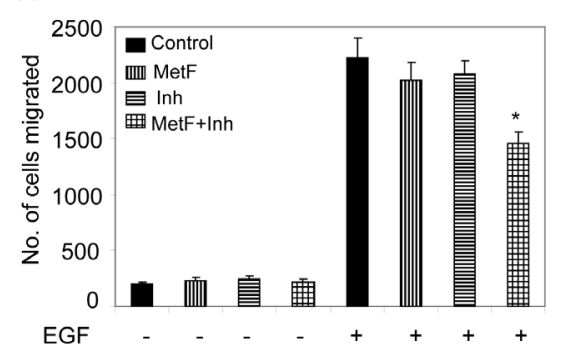

C

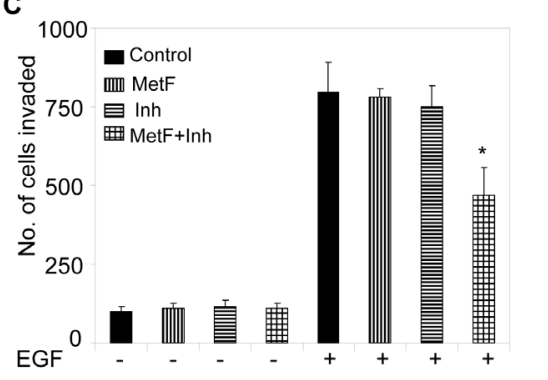

E
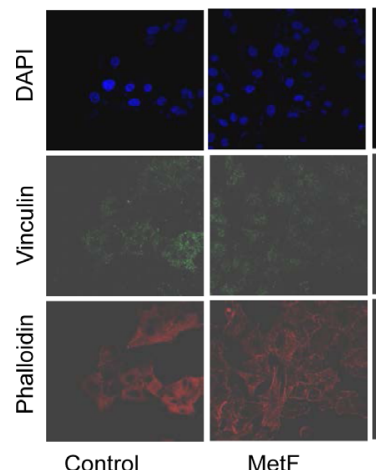

MetF
B

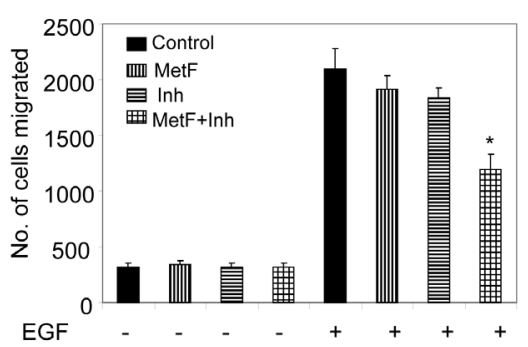

D

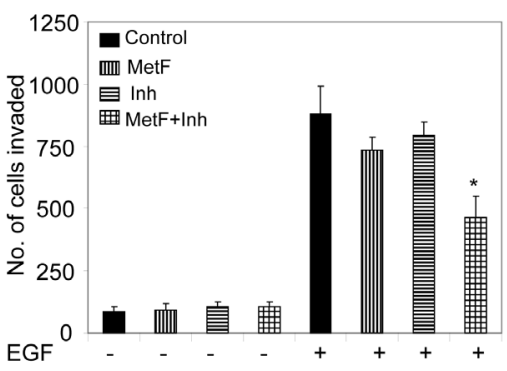

$\mathbf{F}$

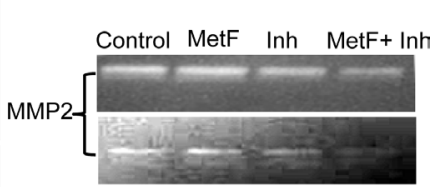

Figure 3: FAAH inhibition enhances the anti-migratory and anti-invasive activities of Met-F-AEA in NSCLC cell lines. A549 (A) and H460 (B) cells were treated with control, Met-F-AEA (MetF, 10 $\mu$ M), FAAH inhibitor URB597 (Inh, $0.2 \mu \mathrm{M})$ or MetF+Inh for $24 \mathrm{~h}$ and subjected to EGF (100ng/ml)-induced migration using transwell plates. The number of cells migrated were stained with Hema stain and counted in five different fields. A549 (C) and H460 (D) cells were treated with control, Met-F-AEA (MetF, 10 $\mu$ M), FAAH inhibitor URB597 (Inh, $0.2 \mu \mathrm{M}$ ) or MetF+Inh for $24 \mathrm{~h}$ and subjected to EGF $(100 \mathrm{ng} / \mathrm{ml}$ )-induced invasion assay using transwell plates coated with matrigel. Invaded cells were stained with Hema stain and counted. (E) Confocal microscopy visualization of A549 cells treated with control, Met-F-AEA (MetF, 10 $\mu \mathrm{M}$ ), FAAH inhibitor URB597 (Inh, 0.2 $\mu \mathrm{M}$ ) or MetF+Inh for 24h and stimulated with EGF (100ng/ml) and stained for phalloidin (red), vinculin (green) expression and DAPI (blue). (F) A549 (upper panel) and H460 (lower panel) cells were treated with control, Met-F-AEA (MetF, 10 $\mu \mathrm{M}$ ), FAAH inhibitor URB597(Inh, 0.2 $\mu \mathrm{M}$ ) or MetF+Inh for 48h and the supernatants were collected, concentrated and run on zymogram gels to detect the active form of MMP2. P $<0.05\left(^{*}\right)$ as calculated by Student's t test. Graphs represent the mean $\pm \mathrm{SD}$ for each experiment repeated three times with similar results. 
and observed significant inhibition in EGF induced cell motility (S1), migration (Fig 3A, B) and invasion (Fig 3C, D) when compared to Met-F-AEA or URB597 alone in A549 and H460 cells.

To migrate through the ECM, cancer cells possess extended protrusions that are rich in actin filaments and adhesion molecules [37]. We investigated the effects of Met-F-AEA when FAAH is inhibited on EGF induced actin stress fiber and focal adhesion formation, which were detected by changes in actin and vinculin expressions, respectively. We observed that cells treated with the combination treatment significantly inhibited EGF induced actin and vinculin expressions more effectively than MetF-AEA or URB597 alone. Further examination with the confocal microscopy revealed the decreased presence of migratory structures such as lamellipodia in the cells treated with the combination treatment when compared to control (Fig 3E).

Tumor invasion begins with protrusion of the ECM and basement membrane. The matrix metalloproteinase (MMP) family has been shown to be responsible for degradation of the ECM, which is one of the initial steps in metastasis. Higher expressions of MMP2 and MMP9 have also been correlated with poor prognosis in early stages of lung adenocarcinoma [38]. In our present study, we observed a significant decrease in secretion of MMP2 by zymogram when cells were treated with combination treatment, Met-F-AEA along with URB597 (Fig 3F). These data suggest that the Met-F-AEA in combination with URB597 inhibits cell migration and invasion in
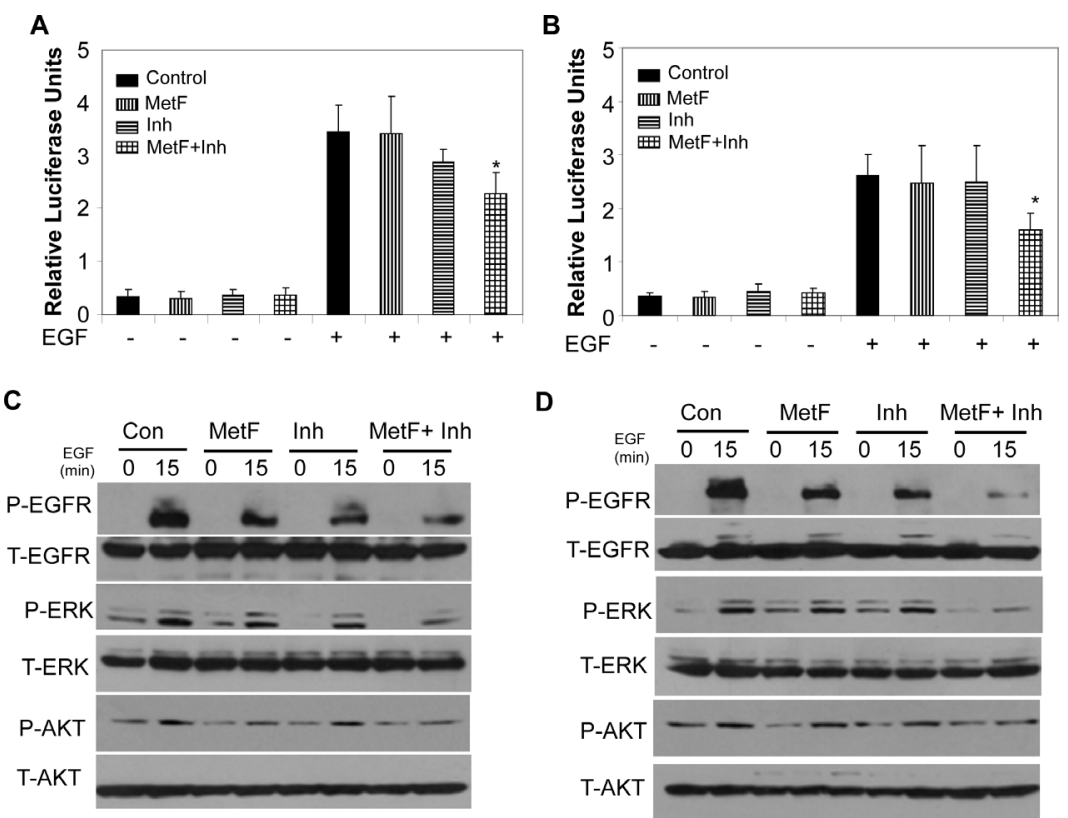

D
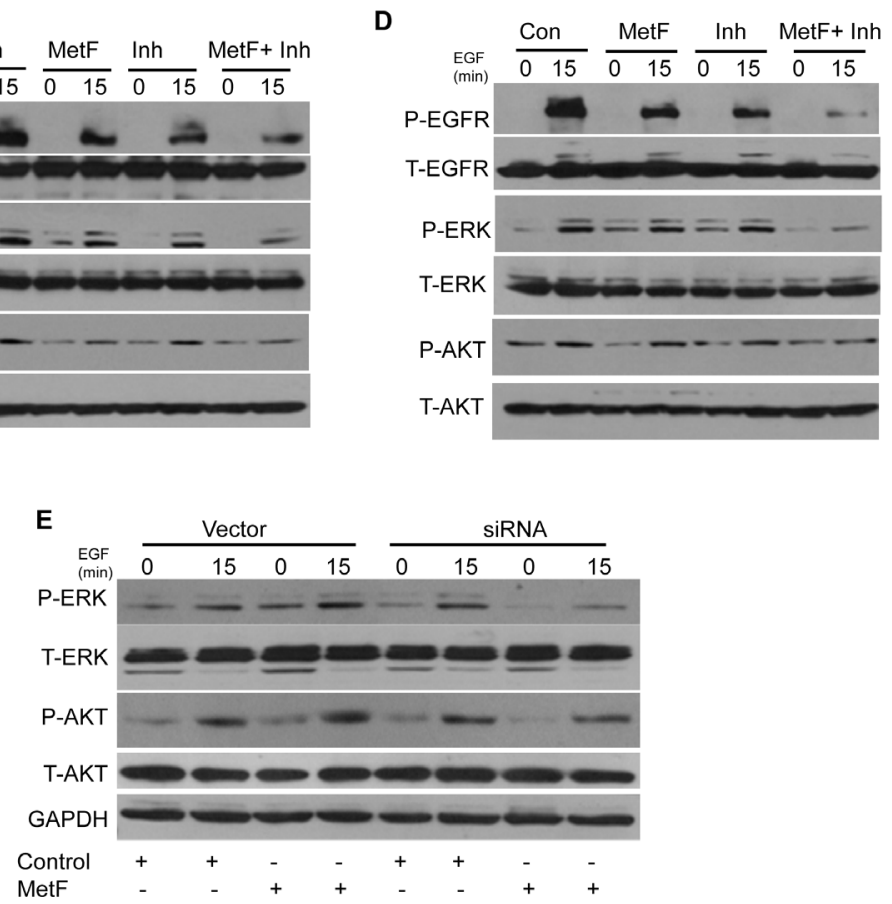

Figure 4: FAAH inhibition enhances Met-F-AEA mediated inhibition of EGFR signaling in NSCLC cell lines. A549 (A) and H460 (B) cells were treated with control, Met-F-AEA (MetF, 10 $\mu$ M), FAAH inhibitor URB597(Inh, 0.2 $\mu$ M) or MetF+Inh for 24h and transfected with either wild-type or NF-kB plasmid for $24 \mathrm{~h}$, stimulated with EGF $(100 \mathrm{ng} / \mathrm{ml})$ for additional $24 \mathrm{~h}$, lysed and analyzed for luciferase activity. Renilla luciferase vector served as internal control. A549 (C) and H460 (D) cells were treated with control, Met-FAEA (MetF, $10 \mu \mathrm{M})$, FAAH inhibitor URB597(Inh, $0.2 \mu \mathrm{M})$ or MetF+Inh for 24h, stimulated with EGF (100ng/ml) for 0 and $15 \mathrm{~min}$ and subjected to Western blot analysis to determine expression of phopho-EGFR, ERK or AKT (P-EGFR, P-ERK, P-AKT) and total EGFR, ERK and AKT (T-EGFR, T-ERK, T-AKT). (E) H460 cells which were transfected with either FAAH siRNA or non targeted control (vector) for $36 \mathrm{~h}$ were treated with either Met-F-AEA or control for $24 \mathrm{~h}$, stimulated with EGF $(100 \mathrm{ng} / \mathrm{ml})$ for 0 and 15 min and analyzed by Western blotting to determine expression levels of phopho-ERK or AKT (P-ERK, P-AKT) and total ERK and AKT (T-ERK, T-AKT). P<0.05 (*) as calculated by Student's t test. Data represent the mean \pm SD for each experiment repeated three times with similar results. 
NSCLC cell lines.

\section{FAAH inhibition enhances Met-F-AEA mediated inhibition of EGFR signaling in NSCLC cell lines}

$\mathrm{NF}-\mathrm{kB}$ is involved in invasion and metastasis in various cancer types [39]. Previous studies have shown that NF-kB activation is involved in EGFR mediated lung tumor progression [39-40]. Therefore, we sought out to determine if FAAH inhibition can enhance Met-F-AEA mediated inhibition of EGF induced NF-kB activation by luciferase reporter assay. Cells were transfected with either vector or NF-kB plasmid and the translocation of NF-kB into the nucleus was studied in the presence or absence of EGF. Met-F-AEA in combination with URB597 treated cells underwent significantly reduced EGF induced NF-kB translocation into the nucleus when compared to Met-FAEA or URB597 alone (Fig 4A,B).

The EGFR signaling pathway activates diverse cellular targets which are crucial for cell proliferation, survival, angiogenesis, migration and adhesion and are often dysregulated in cancer cells. AKT and ERK are important survival molecules that are essential for EGF induced cell growth and motility [22, 25, 32]. To understand the molecular mechanism by which FAAH inhibition enhances Met-F-AEA and regulates EGFR pathway, we treated the cells with Met-F-AEA together with URB597, induced with EGF and observed reduction in the tyrosine phosphorylation of EGFR, serine phosphorylation of AKT and tyrosine phosphorylation of ERK which are the immediate downstream targets
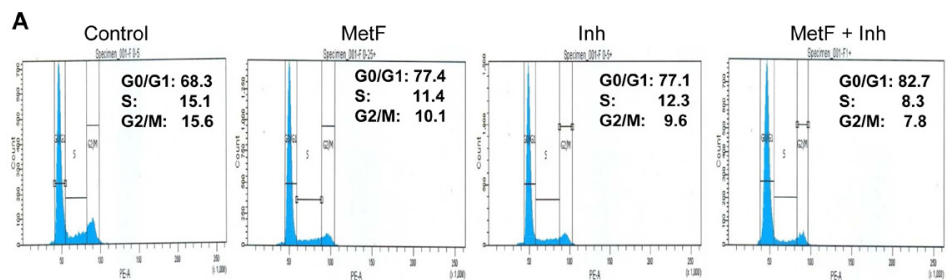

B

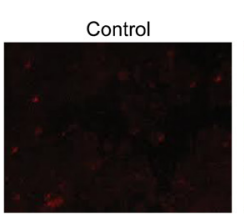

C

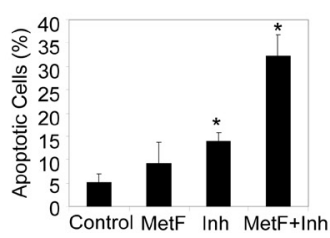

E

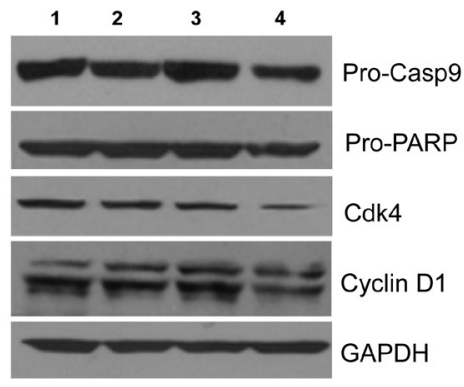

MetF

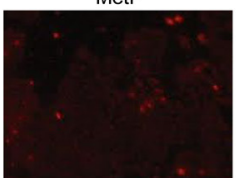

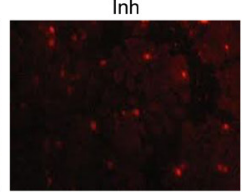

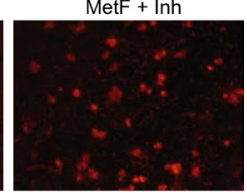

D

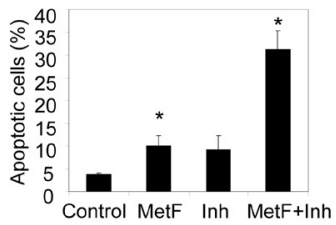

$\mathbf{F}$

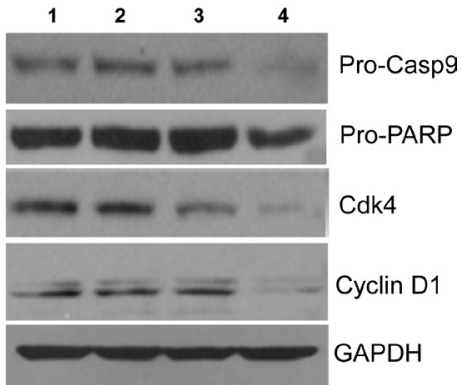

Figure 5: FAAH inhibition enhances Met-F-AEA induced cell cycle arrest and apoptosis at later stage. (A) A549 cells

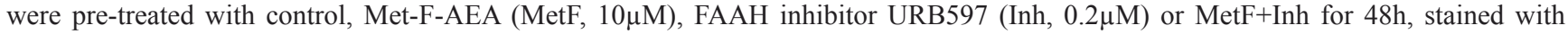
propidium iodide and analyzed for cell cycle arrest by flow cytometry. (B) Representative images of TUNEL positive cells in H460 cells. H460 (C) and A549 (D) cells were pre-treated with control, Met-F-AEA (MetF, 10 $\mu$ M), FAAH inhibitor URB597 (Inh, $0.2 \mu$ M) or MetF+Inh for 48h and subjected to TUNEL assay. The percentage of apoptotic cells was calculated as the percentage of fluorescent positive cells. A549 (E) and H460 (F) cells were pre-treated with control (1), Met-F-AEA (2), FAAH inhibitor URB597(3) or MetF+Inh (4) for $48 \mathrm{~h}$ and subjected to Immunoblot analysis to determine the expression of cell cycle markers cyclin D1, CDK4 and apoptotic markers procaspase-9 and pro-PARP. GAPDH served as loading control. $\mathrm{P}<0.05(*)$ as calculated by Student's $t$ test. Data represent the mean $\pm \mathrm{SD}$ for each experiment repeated three times with similar results. 
of EGFR pathway when compared to Met-F-AEA or URB597 alone (Fig 4C, D).

To further validate that FAAH inhibition enhances the anti-tumorigenic effects of Met-F-AEA, H460 cells were transfected with FAAH siRNA, treated with MetF-AEA or vehicle, stimulated with EGF and analyzed by Western blotting. Cells transfected with FAAH siRNA and treated with Met-F-AEA showed reduced expression levels of P-AKT and P-ERK when compared to Met-FAEA alone (Fig 4E).

Taken together, these results indicate that Met-FAEA in combination with URB597 downregulates EGFR receptor activation and also significantly inhibits the downstream signaling targets of EGFR pathway.

\section{FAAH inhibition enhances Met-F-AEA induced cell cycle arrest and apoptosis at later stage}

Cell cycle dysregulation is frequently associated with cancer growth. EGFR activation leads to tumor cell survival, aberrant cell cycle and evasion of apoptosis, ultimately leading to resistance to cytotoxic therapies $[22,25,32]$. Hence, we sought to assess whether MetF-AEA in combination with URB597 can induce cell cycle arrest leading to apoptosis. Cells were treated with control, Met-F-AEA, URB597 or combination treatment of Met-F-AEA with URB597 for 48h and subjected to cell cycle analysis. We observed significant cell cycle arrest in the $\mathrm{G} 0 / \mathrm{G} 1$ phase in the combination therapy treated
A

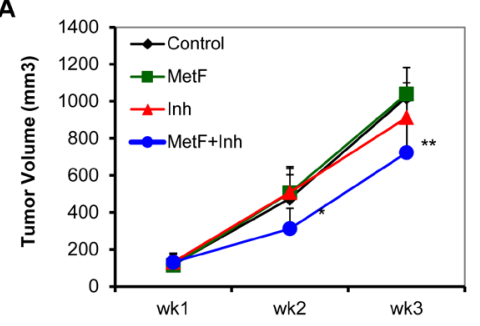

B

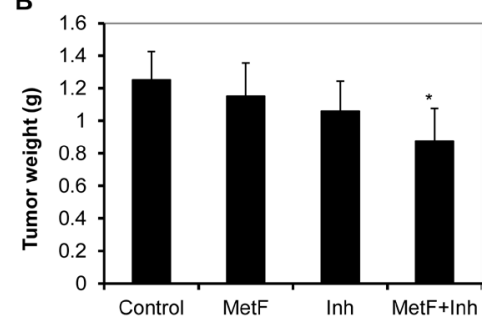

C

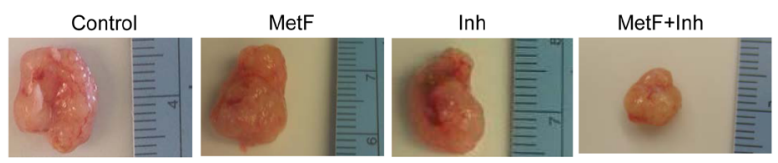

D

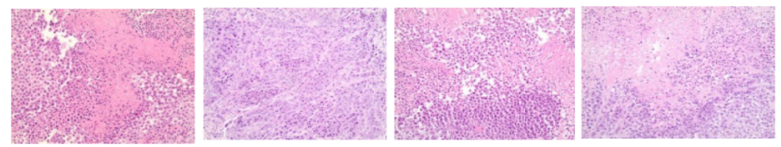

E
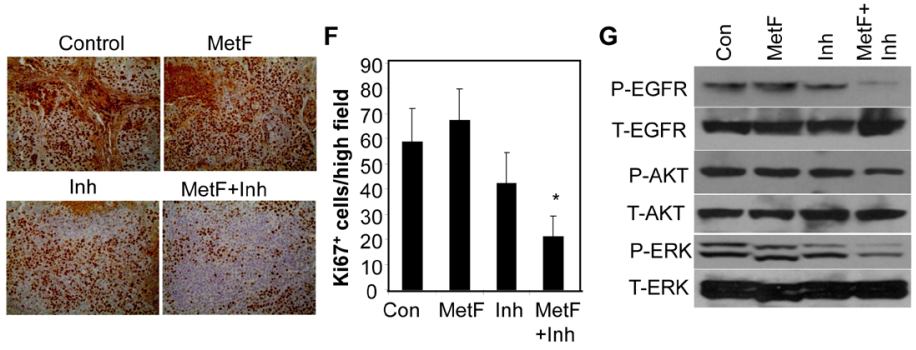

H

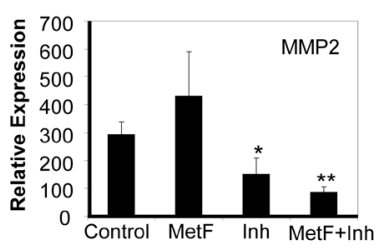

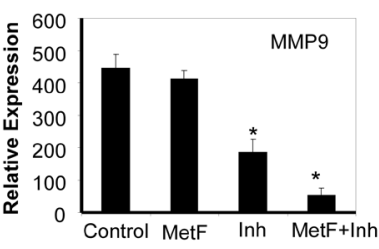

Figure 6: FAAH inhibition enhances Met-F-AEA mediated inhibition of NSCLC tumor growth in vivo by downregulating EGFR signaling. (A) H460 cells were subcutaneously injected in nude mice and palpable tumors were treated with control, Met-F-AEA (MetF, 5mg/kg), FAAH inhibitor URB597 (Inh, 1mg/kg) or MetF+Inh every third day for 3 weeks. Tumor volume was assessed periodically and calculated using the formula $=$ length $\mathrm{x}$ (width) ${ }^{2} / 2$. (B) Tumor weight measured various experimental groups. (C) Representative tumors dissected from various experimental groups. (D) Representative photomicrographs of H\&E and (E) Ki67 staining of tumors extracted from various experimental groups. (F) The number of Ki67 positive cells was counted in four different fields using bright field microscopy in each experimental group and the average was calculated. (G) Xenograft tumors isolated from (A) were subjected to Western blot analysis to determine expression of phospho-EGFR, ERK or AKT (P-EGFR, P-ERK, P-AKT) and total EGFR, ERK and AKT (T-EGFR, T-ERK, T-AKT). Control, Met-F-AEA (MetF), FAAH inhibitor URB597(Inh) or MetF+Inh. (H) Xenograft tumors were subjected to Real Time PCR analysis to determine the expression of MMP2 and MMP9. $\mathrm{P}<0.05(*)$ and $\mathrm{P}<0.005(* *)$ as calculated by Student's t test. Data represent the mean \pm SD for each experiment repeated three times with similar results. 
cells when compared to Met-F-AEA alone (Fig 5A). Met-F-AEA in combination with URB597 increased the percentage of apoptotic cells as shown by TUNEL assay (Fig 5B, C, D) and significantly inhibited pro-caspase9 and pro-PARP. Apoptosis was also induced by cell cycle arrest, as we observed significant decrease in cyclin D1 and CDK4, which are essential for $\mathrm{G} 1 / \mathrm{S}$ phase progression (Fig 5E, F). These results show that Met-F-AEA together with URB597 induced apoptosis and is mediated by cell cycle blockade.

\section{FAAH inhibition enhances Met-F-AEA mediated inhibition of NSCLC tumor growth in vivo by downregulating EGFR signaling}

To evaluate the tumor suppressive effects of Met-FAEA in combination with URB597 in vivo, we determined the anti-tumorigenic potential of the combination treatment on H460 cells in nude mouse model. We induced tumors by injecting $\mathrm{H} 460$ cells subcutaneously into the right flank of male nude mice. When the tumors reached a palpable size, we treated them with ethanol control, MetF-AEA, URB597 or Met-F-AEA in combination with URB597 every third day for three weeks. Tumor volume was monitored throughout the treatment period (Fig 6A). We observed a dramatic decrease in tumor formation in the combination regime treated mice as compared to control (Fig 6B, C). H\&E staining revealed that the combination regime treated tumors were less aggressive and necrotic than the control tumors (Fig 6D). In addition, there was a significant decrease in the expression of proliferation marker Ki67 and hence, a reduced mitotic index in MetF-AEA and URB597 combination treated tumors as compared to Met-F-AEA or URB597 treated tumors alone (Fig 6E, F).

To further determine the mechanism by which the tumors are inhibited, we isolated the tumor xenografts from the nude mice and extracted protein and RNA from them. The Met-F-AEA and URB597 combination treated tumors showed lesser phosphorylation of EGFR, ERK and AKT compared to Met-F-AEA or URB597 treated tumors alone (Fig 6G). Also, real time PCR analysis revealed that MMP2 and MMP9 levels were significantly downregulated in the URB597 treated tumors and Met-FAEA in combination with URB597 treated tumors when compared to the control (Fig 6H), confirming with our in vitro findings.

\section{DISCUSSION}

Lung cancer is estimated to be one of the most fatal cancer related malignancies in the world and fatality is often associated with drug resistance complications. Thus, there is an immediate requirement to develop innovative ways for the treatment of this disease [2-3].
Cannabinoids and their derivatives are being focused on cancer treatments because of their association with pain modulation, cell growth inhibition, anti-inflammation, tumor regression, cell cycle arrest and apoptosis. The endocannabinoid system is involved in a complex array of signaling pathways which might be receptor dependent or independent [7-10]. Though recent studies have shown that endocannabinoids exert potential antitumor effects in various cancer cells [41], not much is known about their effects in lung cancer. Here, we report, for the first time that Met-F-AEA and FAAH inhibitor URB597 significantly inhibited NSCLC growth. We have also explored the mechanisms by which Met-F-AEA in combination with URB597 inhibits NSCLC tumor growth.

In our present study, we found that FAAH and cannabinoid receptor $\mathrm{CB} 1$ are expressed in lung cancer patient samples as well as in NSCLC cell lines. Increased FAAH expression has been associated with poor patient survival in prostate and breast cancer [21, 42]. Also, overexpression of FAAH was found to be sufficient to increase migration and invasion in prostate cancer cells [21]. Our results show that Met-F-AEA that binds to CB1 does not have significant anti-tumorigenic effects in vitro and in vivo. This might be due to the conversion of AEA by enzyme FAAH into metabolites, leading to limitation in the concentration and action of the ligand. Previous reports show that AEA is converted into arachidonic acid (AA) and ethanolamine (EA) by FAAH enzyme [19-20]. AA is metabolized by COX2 and other enzymes to form prostaglandin $\left(\mathrm{PGE}_{2}\right)$ and epoxyeicosatetraenoic acid $(\mathrm{EE})$ [43-44]. These secondary metabolites have been shown to enhance tumor growth and metastasis in various cancer types [44-45]. Also, there are reports which suggest that methanandamide increases murine lung tumor growth by modulation of prostaglandin $\left(\mathrm{PGE}_{2}\right)$ production and $\mathrm{COX} 2$ expression[44]. Therefore, in our study, we blocked FAAH pharmacologically (by using FAAH inhibitor URB597) and genetically (by siRNA approach) to enhance the antitumorigenic effects of Met-F-AEA. Our results show, for the first time, that Met-F-AEA, together with URB597 exerts anti-proliferative effects on NSCLC in vitro and in vivo.

In our study, we have shown that Met-FAEA in combination with URB597 inhibits EGFR phosphorylation and downregulates EGFR mediated signal transduction pathways involving AKT and ERK, which are key cell survival molecules, in vitro and in vivo. This is important because EGF/EGFR axis is known to regulate cancer cells to proliferate and migrate to distant sites. Furthermore, aberrant EGFR expression and function lead to highly aggressive lung tumors, ultimately causing poor patient survival and increased resistance to conventional chemotherapeutic drugs $[22,24]$. Thus, it would be interesting to identify novel targets that have growth inhibiting effects by targeting the EGFR pathway. In our present study, we observed that Met-F-AEA in 
combination with URB597 inhibits stress fiber and focal adhesion formations. Focal adhesions have been shown to connect the ECM to actin stress fibers thus re-organizing the matrix and regulating cell migration [36]. They are the main subcellular macromolecules that form close contacts between cells and the ECM. They play an important role in cell growth and migration. Transformation of epithelial cells into invasive carcinomas also depends on reorganization of the actin cytoskeleton that leads to stress fiber assembly [46]. Furthermore, the Met-F-AEA in combination with URB597 reduces EGF induced invasion and also downregulates MMP2 secretion, which mediates the invasiveness of cancer cells by aiding them to degrade the ECM and metastasize [38].

Our results show that Met-F-AEA in combination with URB597 causes G0/G1 cell cycle arrest mediated apoptosis, which is shown by reduction in G1/S phase checkpoint markers Cyclin D1 and CDK4 and apoptotic markers caspase- 9 and PARP. This is important because in cancer, there exists an imbalance between cell proliferation and apoptosis which leads to tumor progression. Also, uncontrolled cellular growth due to aberrant EGFR signaling leads to dysregulation of the cell cycle, which involves $\mathrm{G} 1 / \mathrm{S}$ checkpoint markers that are responsible for cell cycle progression [26, 47]. Furthermore, apoptosis, a programmed cell death mechanism [48], is usually associated with cell cycle arrest. Hence, our results suggest the ability of Met-F-AEA in combination with URB597 as an apoptosis inducing agent, controlling the cell survival/ death cycle.

Overall, the results of our study suggest that the activity of the endocannabinoid anandamide increases when FAAH is inhibited, leading to enhanced antiproliferative, anti-migratory and anti-invasive effects. We have also shown that Met-F-AEA in combination with URB597 crosstalks with EGF receptor to inhibit its activation, subsequently leading to downregulation of its signaling targets. These implicate that Met-F-AEA along with URB597 can be used as an effective therapeutic strategy for the treatment of EGFR overexpressing NSCLC. This is especially imperative considering the resistance of NSCLC to various chemotherapeutic drugs and its poor prognosis.

\section{MATERIALS AND METHODS}

\section{Reagents and antibodies}

Met-F-AEA and URB597 were purchased from Sigma Aldrich. Antibodies used were P-AKT, caspase-9, PARP, CDK4 (Cell Signaling), P-ERK, ERK, AKT, GAPDH, P-EGFR, EGFR (Santa Cruz), cyclin D1, Ki67 (Neomarkers) and FAAH (Cayman Chemicals).

\section{Cell culture}

Human NSCLC cell lines- A459, A549, CALU1, H1299 and H460 were obtained from ATCC (American Type Culture Collection) and cultured in DMEM or RPMI-1640 (Corning Cellgro), supplemented with 10\% heat inactivated fetal bovine serum (FBS), 5 units $/ \mathrm{mL}$ penicillin, and $5 \mathrm{mg} / \mathrm{mL}$ streptomycin (Corning Cellgro). Cells were maintained at $37^{\circ} \mathrm{C}$ in a humidified $5 \% \mathrm{CO}_{2}$ atmosphere incubator.

\section{Cell proliferation assay}

Cells were seeded at a density of 5000 cells per well in 96 well plates and allowed to grow for 24h. Briefly, cells were treated with Met-F-AEA $(10 \mu \mathrm{M})$, URB597 $(0.2 \mu \mathrm{M})$ or in combination. Cell viability was measured using the MTT assay (Roche) as described in the supplier's protocols, based on the absorbance reading at $570 \mathrm{~nm}$ with respect to the control.

\section{Clonogenic assay}

Cells were seeded at low density in complete media (1000 cells per well in six well plates) and treated with vehicle, Met-F-AEA/URB597 or in combination for six days. After the treatment period, cells were washed with PBS and fixed with $4 \%$ formaldehyde for $20 \mathrm{~min}$, washed again, stained with $0.1 \%$ crystal violet and individual clones were manually counted under the microscope.

\section{Chemotaxis and wound healing assays}

For the migration assay, $8 \mu \mathrm{m}$ transwell plates (Corning-Costar) were used. Briefly, cells were seeded in the upper chamber and chemoattractant EGF (100ng/ml) was added to the lower chambers as previously described. 12 hours after EGF stimulation, cells that migrated to the lower chamber were fixed, stained using Hema stain and counted. For the invasion assay, pre-coated Matrigel invasion chambers (BD Falcon) were used. After 24 hours of stimulation with EGF similar to that in migration assay, invaded cells were stained and counted. Wound scratch assay was done as described previously [49].

\section{FAAH small interfering RNA}

H460 cells were transfected with FAAH siRNA (Dharmacon) using Lipofectamine, as per the manufacturer's recommendations. Scrambled non targeting siRNA was used as control. 36h after transfection, cells were treated with either Met-F-AEA or vehicle and subjected to MTT and western blot analysis. 


\section{Immunofluorescence}

Cells were seeded in 8 well chamber slides, treated, fixed and incubated with primary antibodies phalloidin or vinculin overnight at $4^{\circ} \mathrm{C}$. After washing, cells were stained with Alexa Fluor- 488 or 594 conjugated secondary IgG antibodies and visualized under Olympus FV1000 Filter confocal microscope.

\section{Gelatin zymography}

Gelatin zymography was used to determine MMP activity. Briefly, supernatants of treated cells were collected, concentrated using centrifugal filter units (Millipore) and run on Novex zymogram gel. The gel was then renatured and developed to visualize the bands as per the manufacturer's protocol (Life Technologies).

\section{Luciferase reporter assay}

NF-kB activity was determined using NF-kB luciferase reporter assay (Promega). To determine the luciferase reporter activity, NF-kB luciferase constructs containing either the wild type or NF-kB vector were transfected in the pre-treated cells using lipofectamine. For internal control, cells were co-transfected with Renilla luciferase vector. $24 \mathrm{~h}$ after transfection, EGF $(100 \mathrm{ng} / \mathrm{ml})$ was added and then incubated for another $24 \mathrm{~h}$. Then, the cells were lysed to perform the luciferase assay as per the manufacturer's protocol.

\section{Western blotting}

Cells were washed, lysed and protein estimation was performed using Bradford assay. Aliquots of cellular lysates $(50 \mu \mathrm{g})$ were electorphoresed on a $4-12 \%$ Novex SDS-PAGE, transferred to nitrocellulose membrane and blocked with $5 \%$ non-fat dry milk for an hour at room temperature. The membranes were then probed overnight with specific primary antibody $(1: 1000)$ overnight at $4^{\circ} \mathrm{C}$. After washing thrice with $1 \mathrm{X}$ TBST, blots were exposed to secondary antibody (anti-mouse or anti-rabbit IgG-HRP, 1:2000) for an hour, washed thrice and detected using ECL chemiluminescence detection system (Thermo Scientific).

\section{Cell cycle analysis}

Cells were trypsinized, washed with $1 \mathrm{X}$ PBS and fixed with $70 \%$ ethanol overnight at $4{ }^{\circ} \mathrm{C}$. Then, the cells were spun down, washed twice and incubated with $20 \mu \mathrm{g} /$ $\mathrm{ml}$ propidium iodide and $10 \mu \mathrm{g} / \mathrm{ml}$ RNAse for $30 \mathrm{~min}$, washed and the DNA content was analyzed by flow cytometry.

\section{Apoptosis assay}

Apoptosis was detected in cells using ClickiT TUNEL assay kit (Life Technologies). Cells were washed, fixed and treated with TdT enzyme for $1 \mathrm{~h}$. Then, the cells were stained with AlexaFluor 594 dye-labeled reaction buffer for $30 \mathrm{~min}$ and detected under fluorescent microscope (Olympus).

\section{Mouse xenograft model}

H460 cells $\left(2 \times 10^{6}\right)$ in $100 \mu 1$ PBS were injected subcutaneously into the left flank of each male nude mouse. Once the tumors reached palpable size, they were treated with Met-F-AEA $(5 \mathrm{mg} / \mathrm{kg})$, URB597 $(1 \mathrm{mg} / \mathrm{kg})$ or in combination for 3 weeks. Tumor volume was calculated using the formula vol. $=$ length $*(\text { width })^{2} / 2$.

\section{Real Time PCR}

RNA was isolated from tumors using TriZol reagent (Invitrogen). RT-PCR was performed as described earlier [50].

\section{Tissue Microarray (TMA) and Immunochemical (IHC) analyses}

TMA and IHC were performed as described earlier $[10,50]$.

\section{Statistical analysis}

Results were represented as mean $\pm \mathrm{SD}$ which were analyzed using Student's two-tailed t test. A value of $\mathrm{P}<0.05$ was considered to be statistically significant.

\section{ACKNOWLEDGEMENTS}

The authors thank Susie Jones and Kristin Kovach, Department of Pathology, The Ohio State University, Columbus, Ohio for the immunohistochemical slide analysis of tumor samples. The authors also thank Mustafa Basree for technical help and Dr. Nissar Ahmad Wani and Mohammad Elbaz for reviewing the paper. This work was supported by grants from the NIH (CA163010 and CA153490) and American Lung Association Discovery Award to RKG.

\section{CONFLICTS OF INTEREST}

The authors disclose no competing interests 


\section{REFERENCES}

1. Siegel R, Naishadham D and Jemal A. Cancer statistics, 2013. CA Cancer J Clin. 2013; 63(1):11-30.

2. Salgia R, Hensing T, Campbell N, Salama AK, Maitland M, Hoffman P, Villaflor V and Vokes EE. Personalized treatment of lung cancer. Semin Oncol. 2011; 38(2):274283.

3. West L, Vidwans SJ, Campbell NP, Shrager J, Simon GR, Bueno R, Dennis PA, Otterson GA and Salgia R. A novel classification of lung cancer into molecular subtypes. PLoS One. 2012; 7(2):e31906.

4. Guzman M. Cannabinoids: potential anticancer agents. Nat Rev Cancer. 2003; 3(10):745-755.

5. Matsuda LA, Lolait SJ, Brownstein MJ, Young AC and Bonner TI. Structure of a cannabinoid receptor and functional expression of the cloned cDNA. Nature. 1990; 346(6284):561-564.

6. Munro S, Thomas KL and Abu-Shaar M. Molecular characterization of a peripheral receptor for cannabinoids. Nature. 1993; 365(6441):61-65.

7. Hermanson DJ and Marnett LJ. Cannabinoids, endocannabinoids, and cancer. Cancer Metastasis Rev. 2011; 30(3-4):599-612.

8. Pisanti S, Malfitano AM, Grimaldi C, Santoro A, Gazzerro $\mathrm{P}$, Laezza $\mathrm{C}$ and Bifulco $\mathrm{M}$. Use of cannabinoid receptor agonists in cancer therapy as palliative and curative agents. Best Pract Res Clin Endocrinol Metab. 2009; 23(1):117131.

9. Preet A, Qamri Z, Nasser MW, Prasad A, Shilo K, Zou X, Groopman JE and Ganju RK. Cannabinoid receptors, CB1 and CB2, as novel targets for inhibition of non-small cell lung cancer growth and metastasis. Cancer Prev Res (Phila). 2011; 4(1):65-75.

10. Qamri Z, Preet A, Nasser MW, Bass CE, Leone G, Barsky SH and Ganju RK. Synthetic cannabinoid receptor agonists inhibit tumor growth and metastasis of breast cancer. Mol Cancer Ther. 2009; 8(11):3117-3129.

11. Alexander A, Smith PF and Rosengren RJ. Cannabinoids in the treatment of cancer. Cancer Lett. 2009; 285(1):6-12.

12. Bifulco M, Laezza C, Pisanti $\mathrm{S}$ and Gazzerro $\mathrm{P}$. Cannabinoids and cancer: pros and cons of an antitumour strategy. Br J Pharmacol. 2006; 148(2):123-135.

13. Bifulco M, Laezza C, Portella G, Vitale M, Orlando P, De Petrocellis L and Di Marzo V. Control by the endogenous cannabinoid system of ras oncogene-dependent tumor growth. FASEB J. 2001; 15(14):2745-2747.

14. Bifulco M and Di Marzo V. Targeting the endocannabinoid system in cancer therapy: a call for further research. Nat Med. 2002; 8(6):547-550.

15. Munson AE, Harris LS, Friedman MA, Dewey WL and Carchman RA. Antineoplastic activity of cannabinoids. J Natl Cancer Inst. 1975; 55(3):597-602.

16. Di Marzo V and Maccarrone M. FAAH and anandamide: is
2-AG really the odd one out? Trends Pharmacol Sci. 2008; 29(5):229-233.

17. Sugiura T, Kobayashi Y, Oka S and Waku K. Biosynthesis and degradation of anandamide and 2-arachidonoylglycerol and their possible physiological significance. Prostaglandins Leukot Essent Fatty Acids. 2002; 66(2-3):173-192.

18. Yates ML and Barker EL. Inactivation and biotransformation of the endogenous cannabinoids anandamide and 2-arachidonoylglycerol. Mol Pharmacol. 2009; 76(1):11-17.

19. Cravatt BF, Giang DK, Mayfield SP, Boger DL, Lerner RA and Gilula NB. Molecular characterization of an enzyme that degrades neuromodulatory fatty-acid amides. Nature. 1996; 384(6604):83-87.

20. Wei BQ, Mikkelsen TS, McKinney MK, Lander ES and Cravatt BF. A second fatty acid amide hydrolase with variable distribution among placental mammals. J Biol Chem. 2006; 281(48):36569-36578.

21. Endsley MP, Thill R, Choudhry I, Williams CL, KajdacsyBalla A, Campbell WB and Nithipatikom K. Expression and function of fatty acid amide hydrolase in prostate cancer. Int J Cancer. 2008; 123(6):1318-1326.

22. Seshacharyulu P, Ponnusamy MP, Haridas D, Jain M, Ganti AK and Batra SK. Targeting the EGFR signaling pathway in cancer therapy. Expert Opin Ther Targets. 2012; 16(1):15-31.

23. Mitsudomi $\mathrm{T}$ and Yatabe $\mathrm{Y}$. Epidermal growth factor receptor in relation to tumor development: EGFR gene and cancer. FEBS J. 2010; 277(2):301-308.

24. Zhang Z, Stiegler AL, Boggon TJ, Kobayashi S and Halmos B. EGFR-mutated lung cancer: a paradigm of molecular oncology. Oncotarget. 2010; 1(7):497-514.

25. Preet A, Ganju RK and Groopman JE. Delta9Tetrahydrocannabinol inhibits epithelial growth factorinduced lung cancer cell migration in vitro as well as its growth and metastasis in vivo. Oncogene. 2008; 27(3):339346.

26. Jacks $\mathrm{T}$ and Weinberg RA. Cell-cycle control and its watchman. Nature. 1996; 381(6584):643-644.

27. Sanchez I and Dynlacht BD. New insights into cyclins, CDKs, and cell cycle control. Semin Cell Dev Biol. 2005; 16(3):311-321.

28. Sarfaraz S, Afaq F, Adhami VM, Malik A and Mukhtar H. Cannabinoid receptor agonist-induced apoptosis of human prostate cancer cells LNCaP proceeds through sustained activation of ERK1/2 leading to G1 cell cycle arrest. J Biol Chem. 2006; 281(51):39480-39491.

29. Herrera B, Carracedo A, Diez-Zaera M, Gomez del Pulgar T, Guzman M and Velasco G. The CB2 cannabinoid receptor signals apoptosis via ceramide-dependent activation of the mitochondrial intrinsic pathway. Exp Cell Res. 2006; 312(11):2121-2131.

30. Maccarrone M and Finazzi-Agro A. The endocannabinoid system, anandamide and the regulation of mammalian cell 
apoptosis. Cell Death Differ. 2003; 10(9):946-955.

31. Shrivastava A, Kuzontkoski PM, Groopman JE and Prasad A. Cannabidiol induces programmed cell death in breast cancer cells by coordinating the cross-talk between apoptosis and autophagy. Mol Cancer Ther. 2011; 10(7):1161-1172.

32. Brambilla E and Gazdar A. Pathogenesis of lung cancer signalling pathways: roadmap for therapies. Eur Respir J. 2009; 33(6):1485-1497.

33. De Petrocellis L, Melck D, Palmisano A, Bisogno T, Laezza C, Bifulco M and Di Marzo V. The endogenous cannabinoid anandamide inhibits human breast cancer cell proliferation. Proc Natl Acad Sci U S A. 1998; 95(14):8375-8380.

34. Ligresti A, Bisogno T, Matias I, De Petrocellis L, Cascio MG, Cosenza V, D’Argenio G, Scaglione G, Bifulco M, Sorrentini I and Di Marzo V. Possible endocannabinoid control of colorectal cancer growth. Gastroenterology. 2003; 125(3):677-687.

35. Mimeault M, Pommery N, Wattez N, Bailly C and Henichart JP. Anti-proliferative and apoptotic effects of anandamide in human prostatic cancer cell lines: implication of epidermal growth factor receptor down-regulation and ceramide production. Prostate. 2003; 56(1):1-12.

36. Yamaguchi $\mathrm{H}$, Wyckoff $\mathrm{J}$ and Condeelis J. Cell migration in tumors. Curr Opin Cell Biol. 2005; 17(5):559-564.

37. Yamaguchi $\mathrm{H}$ and Condeelis J. Regulation of the actin cytoskeleton in cancer cell migration and invasion. Biochim Biophys Acta. 2007; 1773(5):642-652.

38. Shou Y, Hirano T, Gong Y, Kato Y, Yoshida K, Ohira T, Ikeda N, Konaka C, Ebihara Y, Zhao F and Kato H. Influence of angiogenetic factors and matrix metalloproteinases upon tumour progression in non-smallcell lung cancer. Br J Cancer. 2001; 85(11):1706-1712.

39. Cai Z, Tchou-Wong KM and Rom WN. NF-kappaB in lung tumorigenesis. Cancers (Basel). 2011; 3(4):4258-4268.

40. Bivona TG, Hieronymus H, Parker J, Chang K, Taron M, Rosell R, Moonsamy P, Dahlman K, Miller VA, Costa C, Hannon G and Sawyers CL. FAS and NF-kappaB signalling modulate dependence of lung cancers on mutant EGFR. Nature. 2011; 471(7339):523-526.

41. Pacher P, Batkai S and Kunos G. The endocannabinoid system as an emerging target of pharmacotherapy. Pharmacol Rev. 2006; 58(3):389-462.

42. Shubbar E, Helou K, Kovacs A, Nemes S, Hajizadeh S, Enerback $\mathrm{C}$ and Einbeigi Z. High levels of gamma-glutamyl hydrolase $(\mathrm{GGH})$ are associated with poor prognosis and unfavorable clinical outcomes in invasive breast cancer. BMC Cancer. 2013; 13:47.

43. Panigrahy D, Edin ML, Lee CR, Huang S, Bielenberg DR, Butterfield CE, Barnes CM, Mammoto A, Mammoto T, Luria A, Benny O, Chaponis DM, Dudley AC, Greene ER, Vergilio JA, Pietramaggiori G, et al. Epoxyeicosanoids stimulate multiorgan metastasis and tumor dormancy escape in mice. J Clin Invest. 2012; 122(1):178-191.
44. Woodward DF, Liang Y and Krauss AH. Prostamides (prostaglandin-ethanolamides) and their pharmacology. $\mathrm{Br}$ J Pharmacol. 2008; 153(3):410-419.

45. Guindon $\mathrm{J}$ and Hohmann AG. A physiological role for endocannabinoid-derived products of cyclooxygenase2-mediated oxidative metabolism. Br J Pharmacol. 2008; 153(7):1341-1343.

46. Friedl $\mathrm{P}$ and Wolf K. Tumour-cell invasion and migration: diversity and escape mechanisms. Nat Rev Cancer. 2003; 3(5):362-374.

47. Malumbres $\mathrm{M}$ and Barbacid M. Mammalian cyclindependent kinases. Trends Biochem Sci. 2005; 30(11):630641.

48. Alenzi FQ. Links between apoptosis, proliferation and the cell cycle. Br J Biomed Sci. 2004; 61(2):99-102.

49. Deol YS, Nasser MW, Yu L, Zou X and Ganju RK. Tumorsuppressive effects of psoriasin (S100A7) are mediated through the beta-catenin/ $\mathrm{T}$ cell factor 4 protein pathway in estrogen receptor-positive breast cancer cells. J Biol Chem. 2011; 286(52):44845-44854.

50. Nasser MW, Qamri Z, Deol YS, Ravi J, Powell CA, Trikha P, Schwendener RA, Bai XF, Shilo K, Zou X, Leone G, Wolf R, Yuspa SH and Ganju RK. S100A7 enhances mammary tumorigenesis through upregulation of inflammatory pathways. Cancer Res. 2012; 72(3):604-615. 